\title{
Tratamento clínico da neurorretinite subaguda difusa unilateral com albendazol
}

\author{
Clinical treatment of diffuse unilateral subacute neuroretinitis with albendazole
}

\author{
Marcus Rudolph Malaguido ${ }^{1}$ \\ Antonio Marcelo Barbante Casella ${ }^{2}$ \\ Daniela Rossetto Garcia Malaguido ${ }^{3}$
}

\begin{tabular}{|l|}
\hline RESUMO \\
\hline Objetivo: Descrever a evolução de uma série de casos de neurorretinite \\
subaguda difusa unilateral (NSDU) tratados com albendazol. Métodos: \\
Relato de série de casos intervencionista. Os autores desenvolveram \\
protocolo de ensaio clínico não controlado, para estudar a evolução \\
clínica de casos de neurorretinite subaguda difusa unilateral tratados com \\
albendazol. Segundo os critérios do protocolo, foram selecionados seis \\
pacientes até o momento desta publicação, que serão descritos separada- \\
mente. Resultados: Dos seis pacientes estudados, quatro apresentavam \\
larva. Todos os seis pacientes tratados com a droga anti-helmíntica \\
apresentaram melhora da acuidade visual e das lesões coriorretinianas \\
multifocais. As larvas identificadas nos pacientes foram inativadas com \\
o tratamento. Nenhum efeito colateral foi observado. Conclusões: A \\
terapia anti-helmíntica com albendazol parece ser benéfica e segura para \\
pacientes com neurorretinite subaguda difusa unilateral. Mais estudos \\
são necessários para avaliar a eficácia do albendazol no tratamento da \\
neurorretinite subaguda difusa unilateral.
\end{tabular}

Descritores: Retinite/quimioterapia; Albendazol/uso terapeutico; Infecções oculares parasitárias; Retina/parasitologia; Baixa visão; Acuidade visual

\section{INTRODUÇÃO}

A neurorretinite subaguda difusa unilateral (NSDU) é uma síndrome descrita por Gass e Braustein ${ }^{(1)}$ que acomete crianças e adultos jovens, caracterizada, precocemente, por perda visual, vitreíte, papilite e agrupamento de lesões branco-acinzentadas na retina, assim como, tardiamente, por progressiva perda visual, atrofia óptica, estreitamento de vasos retinianos e degeneração difusa do epitélio pigmentar. A larva de um nematóide, que ainda não tem sua espécie comprovada com exatidão, tem sido relatada como agente etiológico da NSDU ${ }^{(1-7)}$. Segundo Gass e Braustein, nematóides de dois diferentes tamanhos $(400-1000 \mu \mathrm{m}$ e $1500-2000 \mu \mathrm{m})$ foram encontrados na doença ${ }^{(1)}$. A variante menor poderia ser Toxocara canis $^{(3,6)}$ ou Ancylostoma caninum $^{(7)}$. E Baylisascaris procyonis pode ser a maior larva ${ }^{(4-5)}$.

No Brasil, a primeira descrição da doença foi realizada por Oliveira e Oréfice em sete casos de NSDU presumíveis ${ }^{(8)}$. A primeira identificação da larva foi feita por Souza e Cunha, em 1992(9); e, em 1995, de Souza e Nakashima retiraram o nematóide sub-retiniano e observaram que o mesmo era compatível com o estágio terciário da larva de Toxocara canis pelo seu tamanho e morfologia ${ }^{(3)}$.

Sinais precoces de NSDU são freqüentemente confundidos com coroidite multifocal, epiteliopatia placóide pigmentar multifocal posterior, síndrome dos pontos brancos evanescentes, ou outras neurites ópticas não 
específicas e papilites ${ }^{(6)}$. Entre estes sinais precoces estão: vitreíte, papilite, vasculite retiniana e lesões multifocais envolvendo as camadas externas da retina ${ }^{(1)}$. Sinais tardios incluem escaras coriorretinianas multifocais, degenerações difusas no epitélio pigmentar, estreitamento das artérias retinianas e atrofia óptica; as quais são suficientemente características que o diagnóstico de NSDU pode ser presumido na primeira consulta ${ }^{(6)}$.

Há relatos de neurorretinite subaguda difusa bilateral, com larvas documentadas em ambos os olhos, que foram tratados com laser e obtiveram melhora na acuidade visual bilateral ${ }^{(6,10)}$. Também foi relatado um caso de NSDU confirmado com duas larvas no mesmo olho, as quais foram fotocoaguladas e o paciente evolui com melhora da função visual ${ }^{(11)}$. Na Venezuela, alguns autores $^{(10)}$ relataram um paciente com larvas sub-retinianas identificadas no mesmo olho em momentos diferentes (intervalo de um ano após fotocoagulação da primeira larva).

A conduta na NSDU inclui diagnóstico precoce, quando possível, e destruição da larva por fotocoagulação a laser ${ }^{(12-14)}$; entretanto a localização do nematóide é difícil e ocorre em poucos casos, portanto um tratamento com anti-helmíntico torna-se necessário ${ }^{(12)}$.

Os anti-helmínticos benzimidazóis possuem amplo espectro e têm, em comum, um sistema de anel bicíclico no qual o benzeno se funde nas posições 4 e 5 do heterociclo ${ }^{(15)}$. Nesta classe de antiparasitários estão o tiabendazol (2-[4'-thiazolyl]-benzimidazole), o mebendazol (methyl 5-[benzoyl] benzimidazole-2-carbamate) e o albendazol (methyl 5-[propylthio] benzimidazole-2- carbamate), além de outros não comumente usados em seres humanos ${ }^{(15)}$.

O mecanismo de ação primário dos benzimidazóis envolve sua interação com o citoesqueleto eucariótico da proteína tubulina, inibindo sua polimerização em microtúbulos. A tubulina também está presente nos hospedeiros mamíferos, mas os benzimidazóis parecem apresentar seletividade para as tubulinas dos parasitas, deste modo manifestando baixa toxicidade ao homem ${ }^{(15)}$.

Este estudo tem o objetivo de descrever a evolução de uma série de casos de NSDU tratados com albendazol, e demonstrar a involução de larvas sub-retinianas após o tratamento com albendazol.

\section{MÉTODOS}

Tipo de estudo: Relato de série de casos intervencionista. Foram tratados seis pacientes (seis olhos) com neurorretinite subaguda difusa unilateral.

O projeto de pesquisa que gerou este estudo foi analisado e aprovado pelo Comitê de Ética em Pesquisa do Hospital Universitário da Universidade Estadual de Londrina (UEL). Os pacientes incluídos neste estudo foram instruídos pelos autores a respeito da patologia em questão e do tratamento proposto, e também assinaram um Termo de Consentimento aprovado pelo Comitê de Ética em Pesquisa do Hospital Universitário da UEL.
Critérios de inclusão: Pacientes atendidos no ambulatório de oftalmologia do Hospital Universitário, com diagnóstico de NSDU presumida (quando não se encontra a larva) em fase precoce ou fase tardia (atrofia do nervo óptico e baixa visão); pacientes com diagnóstico de NSDU em fase tardia (já com atrofia do nervo óptico e outros sinais tardios da doença, e baixa visão - prognóstico visual reservado) e presença da larva viva (detectada com retinografias); pacientes que assinarem o termo de consentimento livre e esclarecido.

Critérios de exclusão: Pacientes com diagnóstico de NSDU em fase precoce com a presença da larva (preferência para tratamento com laser); pacientes que não concordaram em participar do projeto e não assinaram o termo de consentimento; pacientes que relataram alergia ao albendazol ou já apresentaram história prévia de reação adversa ao medicamento; pacientes com más condições clínicas gerais; mulheres grávidas ou em amamentação; crianças menores de dois anos de idade.

Os pacientes foram avaliados quanto à idade, gênero, procedência e tempo de queixa principal. Todos foram submetidos a exame oftalmológico completo, incluindo revisão dos antecedentes médicos e oftalmológicos, determinação da melhor acuidade visual corrigida, medida da pressão intraocular e exame biomicroscópico do fundo sob midríase (com lente Volk de 78D).

O diagnóstico de NSDU ativa foi baseado em características clínicas distintas como: persistente inflamação vítrea unilateral, lesões retinianas branco-amareladas ativas, papilite com ou sem vaculite retiniana (estágio precoce). Foi estabelecido o diagnóstico de NSDU fase tardia quando se associava um ou mais dos seguintes achados: atrofia óptica, estreitamento dos vasos retinianos ou degeneração difusa do epitélio pigmentado retiniano.

Os pacientes selecionados fizeram uso do albendazol na posologia de $400 \mathrm{mg} / \mathrm{dia}$, via oral, por um período de 30 dias. Foram orientados a tomar a medicação logo após a principal refeição do dia. Durante o tratamento, os mesmos foram acompanhados semanalmente com exame clínico e oftalmológico completo e também retinografias coloridas para detectar eventual inativação da larva e documentar o comportamento das lesões coriorretinianas multifocais.

Os parâmetros utilizados para registro da evolução clínica foram: A) acuidade visual com correção óptica, medida pela tabela de Snelen; B) redução das lesões coriorretinianas multifocais (documentação feita com retinografias coloridas); e C) involução da larva subretiniana. Todas as medidas foram realizadas antes e após o tratamento.

\section{RESULTADOS}

Usando os critérios acima relacionados, foram selecionados seis pacientes para o tratamento clínico proposto, os quais serão apresentados separadamente.

O caso 6 também foi incluído na série descrita em 2005 da referência número 22 . 


\section{Caso 1}

RF, masculino, 7 anos de idade, procedente de Colombo (Paraná), queixava-se de diminuição da acuidade visual no olho esquerdo há seis meses. A acuidade visual no olho esquerdo era 20/400, e, no olho direito 20/20. O exame dos reflexos pupilares evidenciou defeito pupilar relativo aferente no olho esquerdo. À biomicroscopia apresentava poucas células vítreas no olho acometido, palidez e atrofia da papila no olho esquerdo, degenerações difusas do epitélio pigmentar, assim como lesões coriorretinianas multifocais difusas na região temporal e uma larva sub-retiniana que apresentava movimentação local (Figura 1).

Após 30 dias de tratamento com albendazol, houve diminuição das lesões coriorretinianas multifocais e a larva subretiniana não foi mais encontrada (Figura 2). A acuidade visual do paciente melhorou para 20/200 e, à biomicroscopia, não apresentava células vítreas, mas a palidez de papila se manteve.

\section{Caso 2}

CES, masculino, 9 anos de idade, procedente da região de Londrina (estado do Paraná), com queixa de baixa acuidade visual no olho esquerdo há três anos.

Ao exame apresentava acuidade visual no olho esquerdo de movimentos de mão anteolhos e 20/20 no olho direito, defeito pupilar relativo aferente no olho esquerdo. À fundoscopia apresentou palidez de papila e lesões amareladas difusas coriorretinianas temporais e estreitamento arteriolar retiniano difuso. Não foi encontrado larva.

Após 30 dias de tratamento anti-helmíntico houve melhora

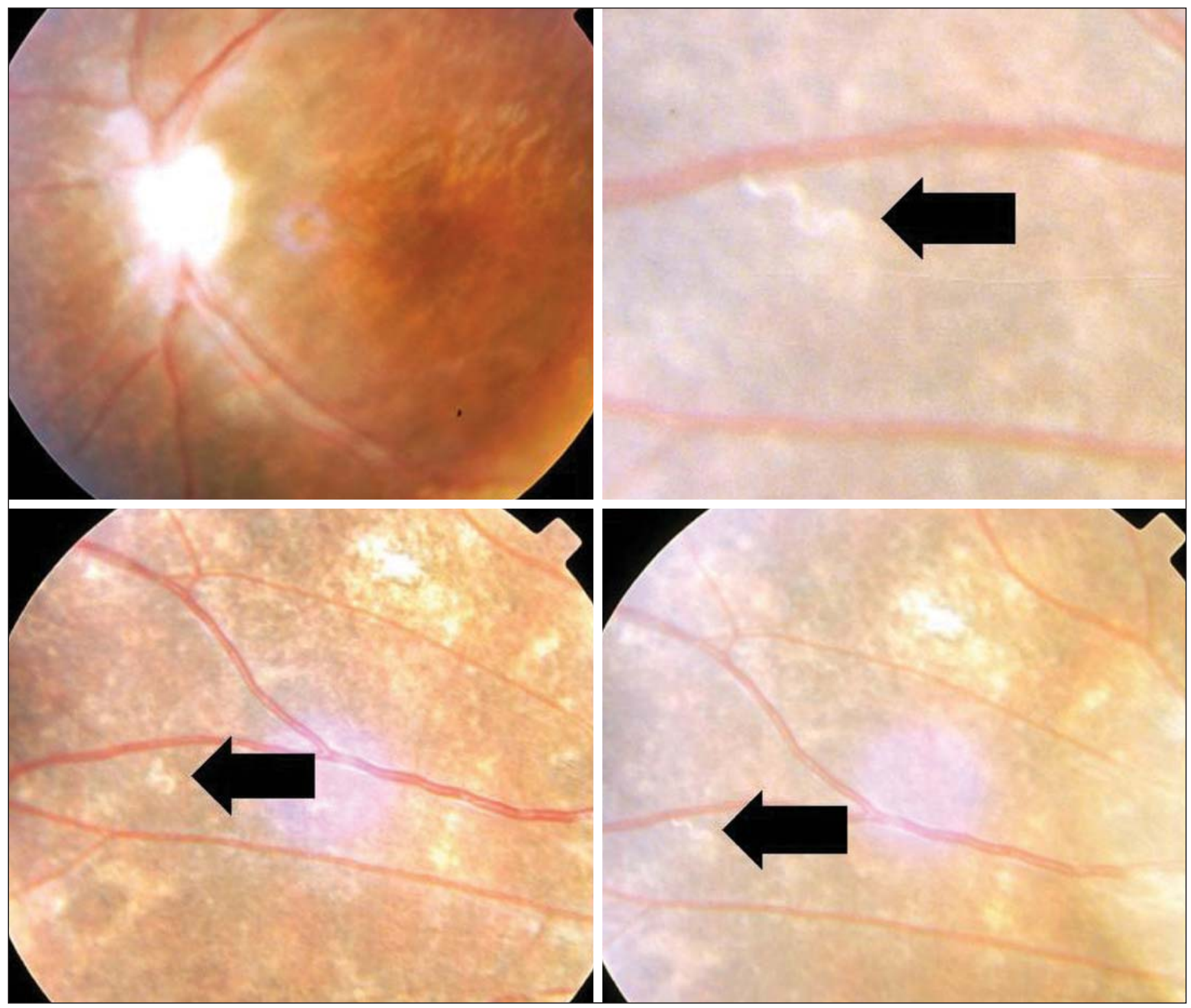

Figura 1 - Paciente 1: Edema de papila, larva com mobilidade (setas) e lesões coriorretinianas 

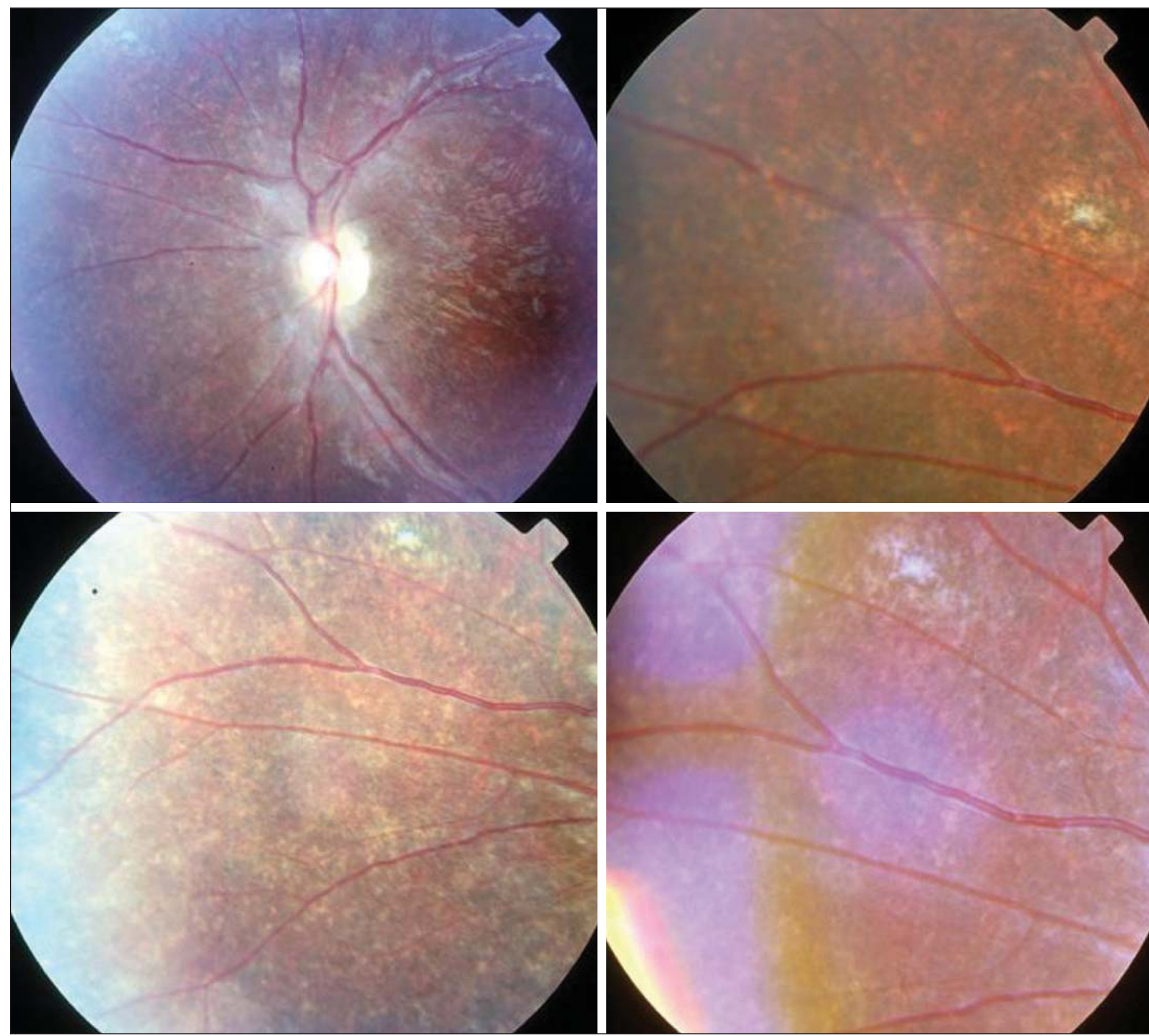

Figura 2 - Paciente 1: Pós-tratamento. Melhora das lesões e do edema de papila e sem larva.

das lesões coriorretinianas e a acuidade visual evoluiu para contagem de dedos a 1 metro. A papila continuou pálida (Figura 3).

\section{Caso 3}

GSN, masculino, 7 anos de idade, procedente da região de Londrina, queixava-se de baixa acuidade visual no olho esquerdo há doze meses. A acuidade visual no olho acometido era 20/400 e no olho direito 20/20. Na biomicroscopia observamos vitreíte, lesões coriorretinianas multifocais, papilite, estreitamento arteriolar retiniano e uma pequena larva móvel.

Após 30 dias de tratamento anti-helmíntico, a acuidade visual evoluiu para 20/70 no olho esquerdo e houve regressão parcial das lesões coriorretinianas multifocais, inativação da larva e melhora da papilite.

\section{Caso 4}

MCMS, feminino, 38 anos de idade, procedente de Londrina, com queixa de diminuição de acuidade visual no olho direito há um ano. A acuidade visual no olho direito era de contagem de dedos a 1 metro e no olho esquerdo 20/20. À fundoscopia apresentava estreitamento vascular, vitreíte e lesões coriorretinianas esbranquiçadas junto à arcada temporal inferior, onde também se evidenciou uma larva.

Após 30 dias de tratamento com albendazol, a acuidade 

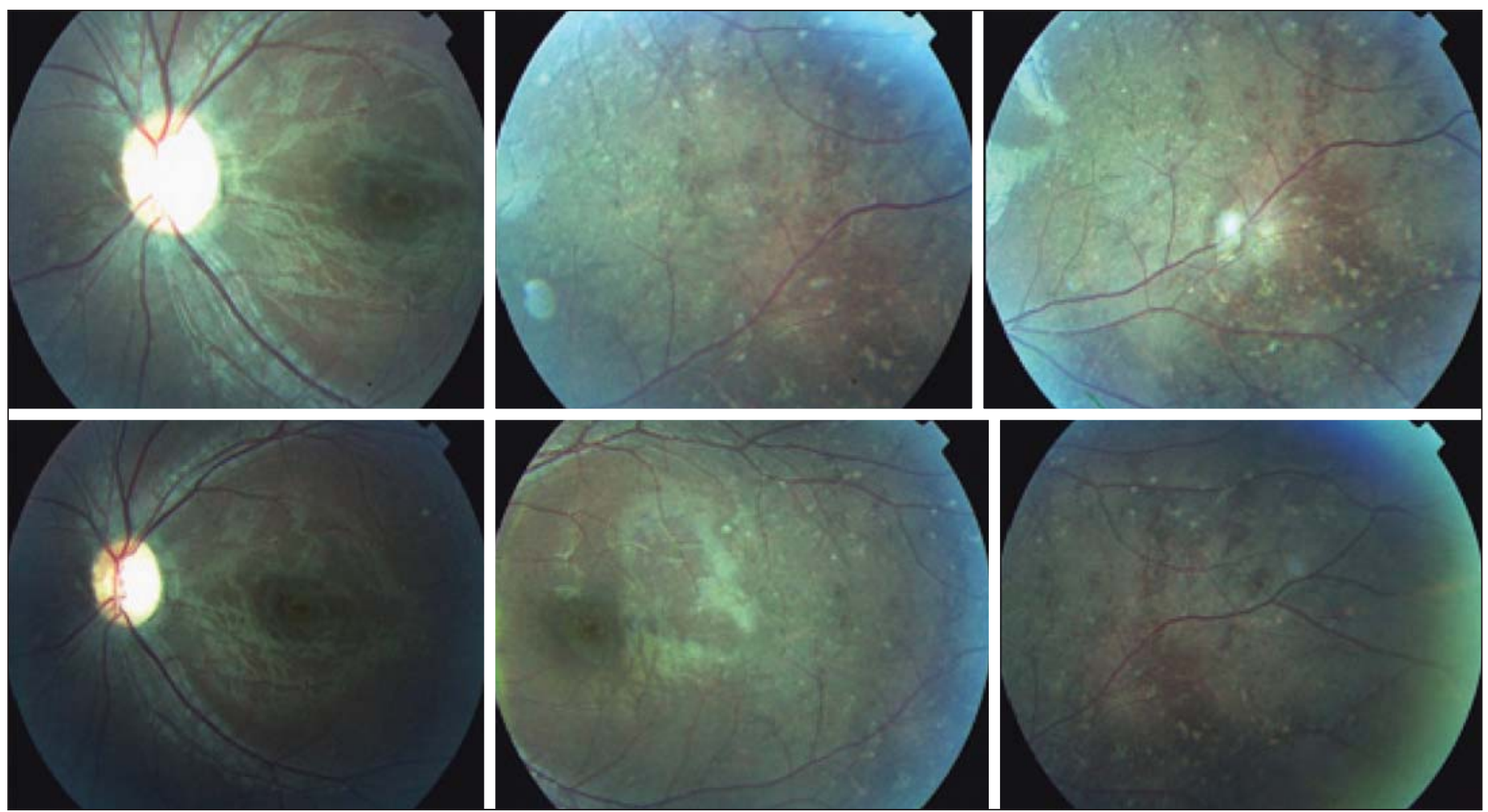

Figura 3 - Paciente 2: Pré-tratamento (superior) e pós-tratamento (inferior)

visual no olho direito era de 20/400, e, ao exame fundoscópico, mostrou involução da larva (Figura 4).

\section{Caso 5}

LA, feminino, 13 anos de idade, procedente de Primeiro de Maio (Paraná), queixava-se de diminuição de acuidade visual no olho esquerdo há um mês. Ao exame apresentava acuidade visual no olho esquerdo de contagem de dedos a 2 metros e, no olho direito 20/20. Ao exame do fundo apresentava lesões coriorretinianas multifocais na região de arcada vascular nasal superior e discreto edema de papila no olho esquerdo. O olho direito não apresentava alteração. $\mathrm{O}$ exame de campimetria visual demonstrou um escotoma absoluto centro-cecal no olho esquerdo. (Figura 5). Não foi encontrado larva.

Após 30 dias de tratamento com albendazol, a acuidade visual evoluiu para 20/25, acompanhada de melhora das lesões coriorretinianas multifocais, do edema de papila e do escotoma centro-cecal (Figura 6).

\section{Caso 6}

MS, masculino, 36 anos de idade, procedente do interior do estado de São Paulo, com queixa de baixa acuidade visual no olho direito há 11 meses.

Ao exame mostrava-se com acuidade visual corrigida de contagem de dedos a $20 \mathrm{~cm}$ no olho direito, defeito pupilar relativo aferente e presença de vitreíte. $\mathrm{O}$ exame do olho esquerdo apresentava-se normal.

O exame biomicroscópico de fundo demonstrou palidez de papila acentuada (atrofia óptica), lesões coriorretinianas difusas branco-amareladas, estreitamento das artérias retinianas, degeneração difusa do epitélio pigmentado retiniano, além da presença de uma larva na região temporal superior à mácula com lesão esbranquiçada ao redor (Figuras 7 e 8).

Após trinta dias de tratamento com albendazol houve melhora das lesões coriorretinianas multifocais e morte com absorção da larva sub-retiniana. A palidez de papila se manteve, e a acuidade visual melhorou para 20/200.

Todos os casos demonstraram estabilidade da acuidade visual e do exame oftalmológico após um ano do tratamento.

Nenhum paciente apresentou reação adversa com o tratamento.

Os dados de todos os pacientes estão resumidos no quadro 1 .

\section{DISCUSSÃO}

O tratamento da NSDU consiste em destruição da larva sub-retiniana ${ }^{(12-14)}$, porém a identificação da mesma ao exame oftalmológico é um grande desafio, e apenas em poucos pacientes isto ocorre ${ }^{(12)}$. Portanto um tratamento com droga anti-helmíntica torna-se necessário.

O tiabendazol e a ivermectina (um macrocíclico) foram usados em alguns casos presumidos de NSDU com resposta favorável ${ }^{(12,16)}$, porém, alguns autores usaram estas drogas em dois casos com larvas vivas e não observaram a morte das larvas, sendo necessário destruí-las através de fotocoagulação a laser ${ }^{(17)}$.

O albendazol possui amplo espectro, comodidade poso- 

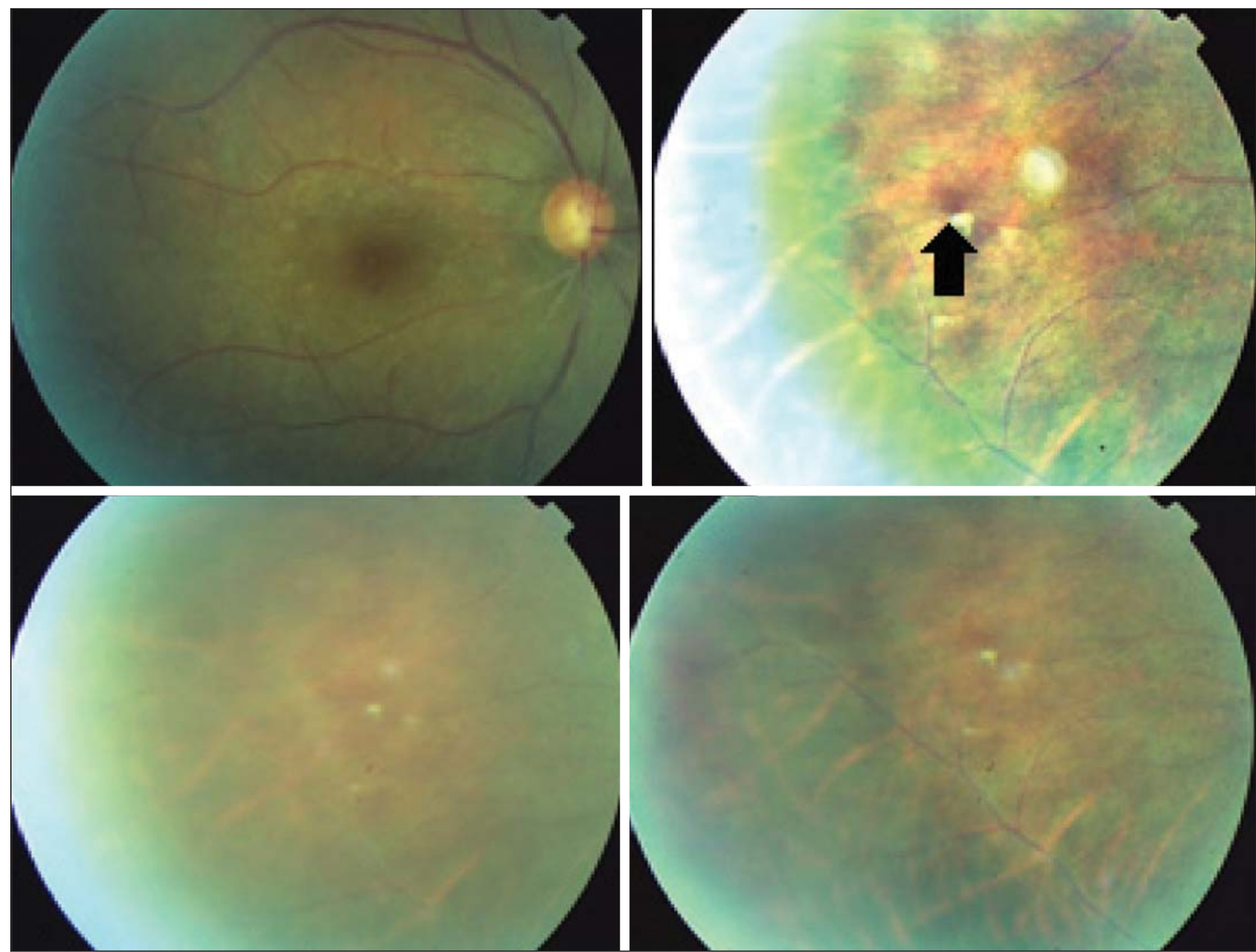

Figura 4 - Paciente 4: Pré-tratamento (superior) e pós-tratamento (inferior). Seta indica a larva.

lógica e baixo índice de efeitos colaterais ${ }^{(15)}$. Foi aprovado para uso em humanos em 1983, e, como o mebendazol, sua principal ação anti-helmíntica é exercida diretamente no trato gastrointestinal. Atinge concentrações plasmáticas 15 a 49 vezes mais altas que o mebendazol, e o seu principal metabólito hepático (o albendazol sulfóxido) também tem ação antihelmíntica ${ }^{(15)}$. A ação anti-helmíntica do albendazol ocorre contra vermes adultos, larvas e ovos ${ }^{(15)}$.

Efeitos adversos do albendazol são raros. Em dosagens usadas para tratamento de helmintíases intestinais já foram relatados: dor epigástrica, diarréia, náuseas e vômitos, cefaléia, vertigem e fenômenos alérgicos ${ }^{(15)}$. Em altas doses e uso prolongado já foram descritos anormalidades na função hepática e alopecia ${ }^{(15)}$.

$\mathrm{O}$ albendazol teve efeito teratogênico em experimentos com ratos e coelhos após alta dosagem, portanto deve ser evitado no primeiro trimestre de gestação ${ }^{(15)}$.

O albendazol, que apresenta boa penetração no sistema nervoso central ${ }^{(18)}$, tem sido usado para combater infecções por larva migrans de Baylisascaris procyonis e Toxocara canis no cérebro e na órbita ${ }^{(19-20)}$; e estas espécies, além de outras, podem ter relação etiológica com a NSDU(3,6).

A melhor dose de albendazol e a duração do tratamento para NSDU ainda não são conhecidas. A escolha deste esquema de trinta dias foi baseado no tratamento da neurocisticercose com albendazol ${ }^{(18,21-22)}$. Nesta série de casos, começamos a observar a melhora dos sinais biomicroscópicos alguns dias antes de completar o trigésimo dia de tratamento.

Em uma série de doze pacientes com diagnóstico de NSDU tratados com albendazol, alguns autores observaram melhora das lesões multifocais e da acuidade visual em todos os pacientes após trinta dias de tratamento com dose de $400 \mathrm{mg} / \mathrm{dia}^{(22)}$. Na Venezuela, outros autores usaram albendazol em seis casos, na dosagem via oral de $200 \mathrm{mg}$ três vezes por dia por dez dias, e observou melhora em apenas três pacientes (inclusive com morte da larva) ${ }^{(10)}$.

Nesta série de casos, associou-se a melhora clínica dos pacientes à involução da larva sub-retiniana (casos 1, 3, 4 e 6 comprovados com a presença da larva, e casos 2 e 5 com larva 


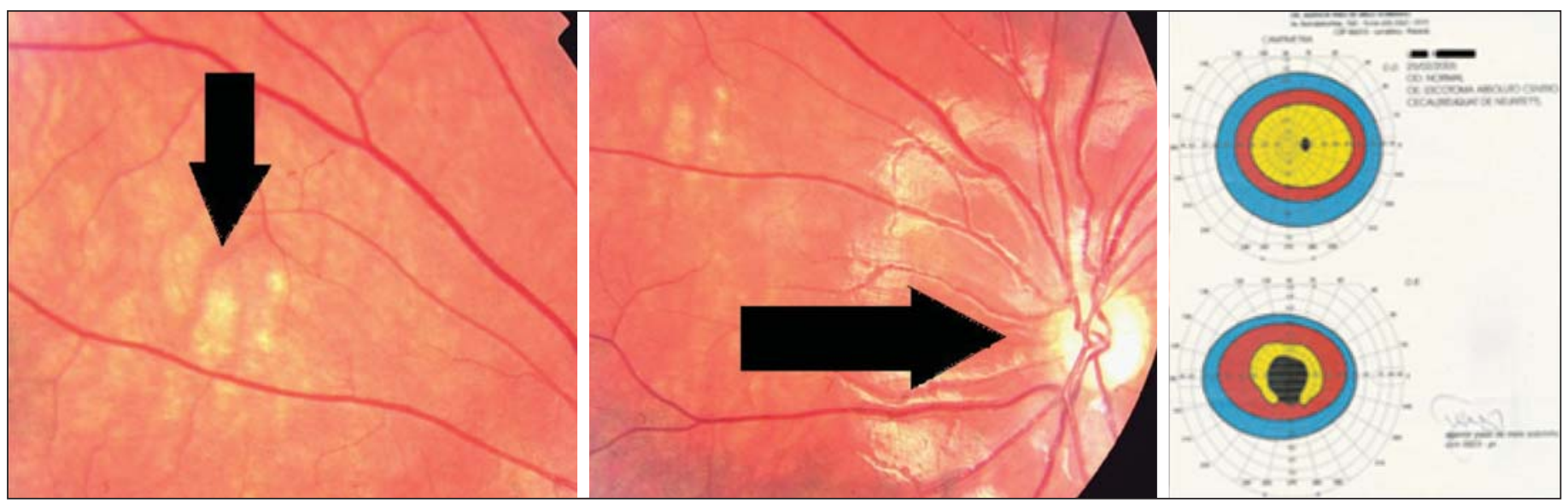

Figura 5 - Paciente 5: Pré-tratamento. Retinografias (lesões coriorretinianas, seta vertical e edema de papila, seta horizontal) e Campimetria.

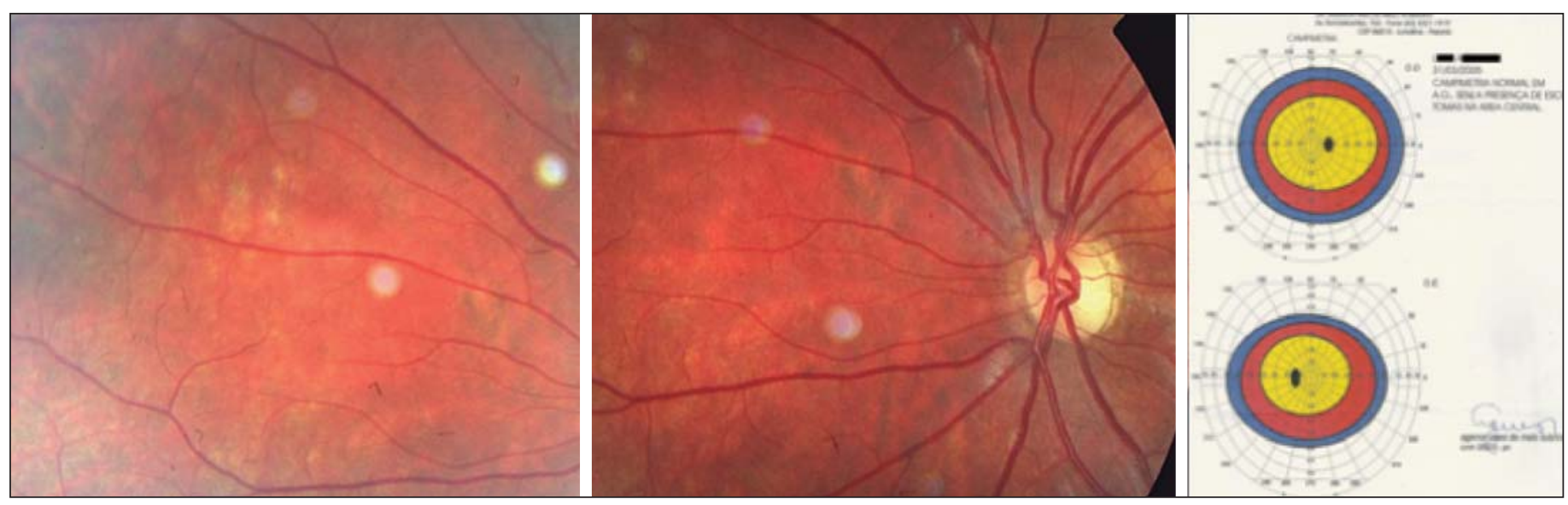

Figura 6 - Paciente 5: Pós-tratamento. Retinografias (melhora das lesões coriorretinianas e do edema de papila) e Campimetria.

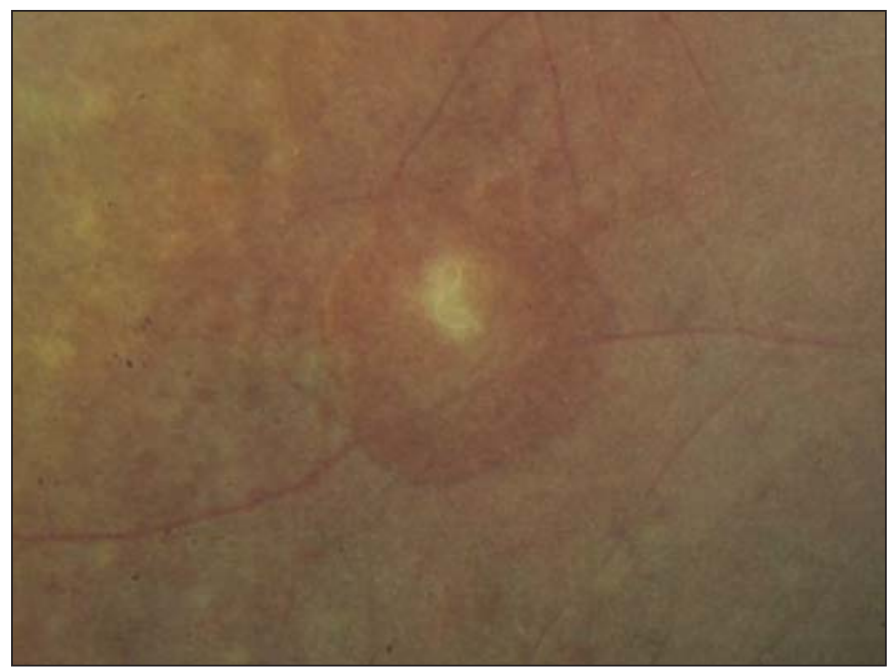

Figura 7 - Paciente 6: Pré-tratamento. Detalhe da larva sub-retiniana (S invertido).

presumida), com conseqüente melhora das lesões coriorretinianas multifocais.

Dos seis pacientes tratados, todos apresentaram melhora da

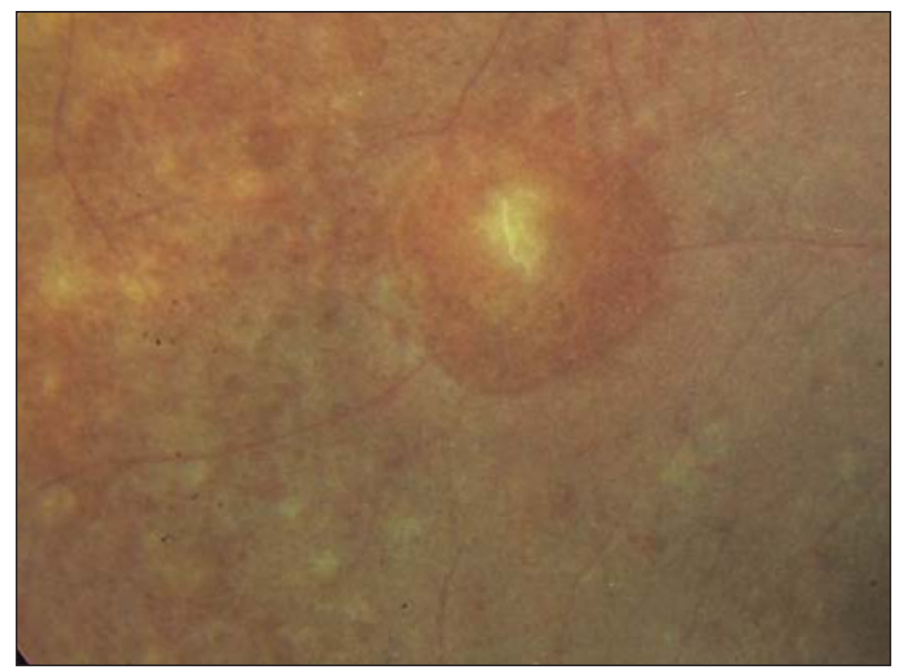

Figura 8 - Paciente 6 em tratamento. Detalhe da larva em destruição e imóvel.

acuidade visual. $\mathrm{O}$ caso 5 foi o que apresentou melhor evolução da acuidade visual (contagem de dedos a 2 metros para 20/25). Isto se deve ao fato de apenas este paciente se apresentar para 


\begin{tabular}{|c|c|c|c|c|c|c|}
\hline Caso & $\begin{array}{l}\text { Idade/ } \\
\text { gênero }\end{array}$ & $\begin{array}{l}\text { Olho/ } \\
\text { fase NSDU }\end{array}$ & $\begin{array}{l}\text { AV pré- } \\
\text { tratamento }\end{array}$ & $\begin{array}{l}\text { Sinais biomicroscópicos } \\
\text { pré-tratamento }\end{array}$ & $\begin{array}{l}\text { AV pós- } \\
\text { tratamento }\end{array}$ & $\begin{array}{l}\text { Sinais biomicroscópicos } \\
\text { pós-tratamento (1 } 1 \text { ês) }\end{array}$ \\
\hline 1 & 7 anos/ M & E/fase tardia & $20 / 400$ & $\begin{array}{l}\text { Papilite, vitreíte+, escaras } \\
\text { coriorretinianas }+++ \text { e uma larva. }\end{array}$ & $20 / 200$ & $\begin{array}{l}\text { Atrofia óptica, vitreíte-, escaras } \\
\text { coriorretinianas+ e sem larva }\end{array}$ \\
\hline 2 & 9 anos/M & $\mathrm{E} / \mathrm{fase}$ tardia & MM & $\begin{array}{l}\text { Atrofia óptica, escaras } \\
\text { coriorretinianas++, estreitamento } \\
\text { arteriolar retiniano. Sem larva visível. }\end{array}$ & $C D$ & $\begin{array}{l}\text { Atrofia óptica, escaras } \\
\text { coriorretinianas+, estreitamento } \\
\text { arteriolar retiniano. }\end{array}$ \\
\hline 3 & 7 anos/M & $\mathrm{E} /$ fase tardia & $20 / 400$ & $\begin{array}{l}\text { Papilite, vitreíte++, escaras } \\
\text { coriorretinianas+++, estreitamento } \\
\text { arteriolar retiniano e uma larva. }\end{array}$ & $20 / 70$ & $\begin{array}{l}\text { Vitreíte+, escaras coriorretinianas-, } \\
\text { estreitamento arteriolar retiniano } \\
\text { e sem larva. }\end{array}$ \\
\hline 4 & 38 anos/F & $\mathrm{D} /$ fase tardia & $C D$ & $\begin{array}{l}\text { Escaras coriorretinianas++, } \\
\text { estreitamento arteriolar, vitreíte++ } \\
\text { e uma larva. }\end{array}$ & $20 / 400$ & $\begin{array}{l}\text { Escaras coriorretinianas-, } \\
\text { estreitamento arteriolar, vitreíte+ } \\
\text { e sem larva. }\end{array}$ \\
\hline 5 & 13 anos/F & E/fase precoce & $C D$ & $\begin{array}{l}\text { Papilite, lesões coriorretinianas ++. } \\
\text { Sem larva visível. }\end{array}$ & $20 / 25$ & $\begin{array}{l}\text { Papila normal, lesões } \\
\text { coriorretinianas- }\end{array}$ \\
\hline 6 & 36 anos/M & $\mathrm{D} /$ fase tardia & $C D$ & $\begin{array}{l}\text { Atrofia óptica, escaras coriorretinia- } \\
\text { nas+++, vitreíte++, estreitamento } \\
\text { arteriolar retiniano e uma larva. }\end{array}$ & $20 / 200$ & $\begin{array}{l}\text { Atrofia óptica, escaras coriorreti- } \\
\text { nianas-, vitreíte+, estreitamento } \\
\text { arteriolar retiniano e sem larva. }\end{array}$ \\
\hline
\end{tabular}

tratamento em fase precoce (queixa de um mês), enquanto todos os outros já tinham queixas que variavam de seis meses a três anos. Logo se aponta a importância do diagnóstico precoce.

Considerando a possibilidade de haver uma infestação por larva migrans em outros órgãos, inclusive no tecido cutâneo $^{(7)}$, ressaltamos a validade do tratamento sistêmico antihelmíntico nos casos suspeitos e confirmados de NSDU. Também enfatizamos a importância deste tratamento nos casos pediátricos, onde o exame detalhado do fundo de olho nem sempre pode ser feito com a colaboração do paciente. Nesta situação o tratamento anti-helmíntico pode trazer comodidade com baixo risco e bons resultados.

\section{CONCLUSÕES}

A terapia anti-helmíntica com albendazol parece ser benéfica e segura para pacientes com NSDU. Mais estudos são necessários para avaliar a eficácia do albendazol no tratamento da neuroretinite subaguda difusa unilateral.

\section{ABSTR ACT}

Purpose: To describe the evolution of a series of cases of diffuse unilateral subacute neuroretinitis (DUSN) treated with albendazole. Methods: Interventional case series. The authors developed a non-randomized clinical trial protocol to investigate the clinical evolution of diffuse unilateral subacute neuroretinitis cases treated with albendazole. According to protocol criteria up to now, six patients were selected that will be described separately. Results: Of the six studied patients, four presented the worm. All six patients treated with the antiparasitic drug showed improvement of visual acuity and of chorioretinal scars. During the weeks of treatment, evidence of worm inactivation was documented for the four patients with visible worms. No adverse drug side effects were observed. Conclusions: The antiparasitic drug albendazole seems to be beneficial and safe in patients with diffuse unilateral subacute neuroretinitis. More studies are necessary to evaluate the effectiveness of albendazole in the treatment of diffuse unilateral subacute neuroretinitis.

Keywords: Retinitis/drug therapy; Albendazole/therapeutic use; Eye infections, parasitic; Retina/parasitology; Low vision; Visual acuity

\section{REFERÊNCIAS}

1. Gass JDM, Braunstein RA. Further observations concerning the diffuse unilateral neuroretinitis syndrome. Arch Ophthalmol. 1983;101(11):1689-97.

2. Casella AM, Bonomo PP, Farah ME, de Souza EC. Diffuse unilateral subacute neuroretinitis (DUSN): 3 cases in Paraná state. Arq Bras Oftalmol. 1994;57(1):77-9.

3. Souza EC, Nakashima Y. Diffuse unilateral subacute neuroretinitis. Report of transvitreal surgical removal of a subretinal nematode. Ophthalmology. 1995; 102(8):1183-6

4. Mets MB, Noble AG, Basti S, Gavin P, Davis AT, Shulman ST, et al. Eye findings of diffuse unilateral subacute neuroretinitis and multiple choroidal infiltrates associated with neural larva migrans due to Baylisascaris procyonis. Am J Ophthalmol. 2003;135(6):888-90.

5. Akao N, Hayashi E, Sato H, Fujita K, Furuoka H. Diffuse retinochoroiditis due to Baylisascaris procyonis in Mongolian gerbils. J Parasitol. 2003;89(1):174-5.

6. Souza EC, Abujamra S, Nakashima Y, Gass JD. Diffuse bilateral subacute neuroretinitis: first patient with documented nematodes in both eyes. Arch Ophthalmol. 1999;117(10):1349-51.

7. Casella AM, Machado RA, Tsuro A, Hato M, Costa R, Farah ME. Seria o Ancylostoma caninum um dos agentes da neuroretinite sub-aguda difusa unilateral (D.U.S.N.) no Brasil? Arq Bras Oftalmol. 2001;64(5):473-6.

8. Oliveira AA, Oréfice F. Estudo de sete casos de neuroretinite difusa subaguda unilateral. Rev Bras Oftalmol. 1991;51(1):51-5.

9. Souza EC, Cunha SL. Neurorretinite sub-aguda unilateral difusa no Brasil: encontro da larva sub-retiniana. Arq Bras Oftalmol. 1992;55(6):251-4.

10. Cortez R, Denny JP, Muci-Mendoza R, Ramirez G, Fuenmayor D, Jaffe GJ. Diffuse unilateral subacute neuroretinitis in Venezuela. Ophthalmology. 2005;112(12):2110-4

11. Harto MA, Rodriguez-Salvador V, Avinó JA, Duch-Samper AM, Menezo JL. Diffuse unilateral subacute neuroretinitis in Europe. Eur J Ophthalmol. 1999;9(1):58-62. 
12. Gass JD, Callanan DG, Bowman B. Oral therapy in diffuse unilateral subacute neuroretinitis. Arch Ophthalmol. 1992;110(5):675-80.

13. Garcia CA, Gomes AH, Garcia Filho CA, Vianna RN. Early-stage diffuse unilateral subacute neuroretinitis: improvement of vision after photocoagulation of the worm. Eye. 2004;18(6):624-7.

14. Martidis A, Greenberg PB, Rogers AH, Velázquez-Estades LJ, Baumal CR. Multifocal electroretinography response after laser photocoagulation of a subretinal nematode. Am J Ophthalmol. 2002;133(3):417-9.

15. Silva N, Guyatt H, Bundy D. Anthelmintics. A comparative review of their clinical pharmacology. Drugs. 1997;53(5):769-88.

16. Callanan DG, Davis JL, Cohen SM, Halperin L, Gass JD. The use of ivermectin in diffuse unilateral subacute neuroretinitis. Ophthalmology. 1993;100(9 Suppl):114-5.

17. Casella AMB, Farah ME, Belfort Jr R. Antihelminthic drugs in diffuse unilateral subacute neuroretinitis. Am J Ophthalmol. 1998;125(1):109-11.
18. Jung H, Hurtado M, Sanchez M, Medina MT, Sotelo J. Plasma and CSF levels of albendazole and praziquantel in patients with neurocysticercosis. Clin Neuropharmacol. 1990;13(6):559-64

19. Dubovskaia LA, Tumol'skaia NI, Sidorenko NI, Kotiasheva GI, Odoshashvili $\mathrm{ED}$, Gorbunov AV. [Clinical course of isolated larval infestation of orbit in children]. Vestn Oftalmol 2000;116(3):37-9. Russian.

20. Murray WJ, Kazacos KR. Raccoon roundworm encephalitis. Clin Infect Dis. 2004;39(10):1484-92.

21. Singhi P, Dayal D, Khandelwal N. One week versus four weeks of albendazole therapy for neurocysticercosis in children: a randomized, placebo controlled double blind trial. Pediatr Infect Dis J. 2003;22(3):268-72.

22. Souza EC, Casella AM, Nakashima Y, Monteiro ML. Clinical features and outcomes of patients with diffuse unilateral subacute neuroretinitis treated with oral albendazole. Am J Ophthalmol. 2005;140(3):437-45. Erratum in: Am J Ophthalmol. 2006;141(4):795-6. 